\title{
Optical study of good quality InGaP/GaAs quantum wells: Influence of the indium content around the lattice-matched composition
}

\author{
Juan Martínez-Pastor \\ Dep. Física Aplicada, Universitat de València, 46100 Burjassot (Valencia), Spain \\ Luisa González \\ Centro Nacional de Microelectrónica, Serrano 144, 28006 Madrid, Spain \\ Philippe Roussignol \\ Lab. de Physique de la Matière Condensée de l'ENS, 24 Rue Lhomond, 75231 Paris Cedex 05, France
}

(Received 30 November 1995; accepted for publication 7 February 1996)

\begin{abstract}
High structural and optical quality $\operatorname{In}_{x} \mathrm{Ga}_{1-x} \mathrm{P} / \mathrm{GaAs}$ quantum wells, with $x$ from 0.51 to 0.45 , have been successfully grown by atomic layer molecular beam epitaxy. In that compositional range, an important blue shift of the quantum well luminescence lines is observed, which is explained by an increase of the conduction band gap offset from compressive to tensile strain conditions. The luminescence intensity decreases with temperature above $20-30 \mathrm{~K}$, which is attributed to impurities located at the interfaces and inside the quantum wells. The influence of the In content on the oscillator strength of the optical transitions is also evaluated. (C) 1996 American Institute of Physics. [S0003-6951(96)04015-5]
\end{abstract}

A great deal of work has been devoted in the last years to the growth, control, and characterization of InGaP epitaxial layers lattice matched to GaAs (Refs. 1-3 and references therein). InGaP layers have been proposed as a partner of $\mathrm{GaAs}$ to be used as a good substitute of $\mathrm{AlGaAs}$ in electronic and optoelectronic devices, ${ }^{4-8}$ since the last one contains a large concentration of DX-centers and has a high reactivity with oxygen. In this letter we present preliminary results on the optical properties and excitonic recombination in highquality $\mathrm{In}_{x} \mathrm{Ga}_{1-x} \mathrm{P} / \mathrm{GaAs}$ quantum wells (QWs), with $x$ ranging from 0.45 to 0.51 .

The structures under study were grown under the same conditions on semi-insulating (001)-GaAs substrates, after a mixed $700+300 \mathrm{~nm}(500+500 \mathrm{~nm}$ in sample 1) thick $\mathrm{GaAs}+\mathrm{In}_{x} \mathrm{Ga}_{1-x} \mathrm{P}$ buffer layer. The GaAs buffer layers were grown at $580{ }^{\circ} \mathrm{C}$ by MBE, while the InGaP buffer layers and the heterostructures by atomic layer MBE (ALMBE) at $420{ }^{\circ} \mathrm{C}$. The phosphorous beam equivalent pressure never exceeded $10^{-6}$ Torr and the growth rate was 1 monolayer per second. The structures consist in five GaAs quantum wells of different nominal thicknesses: 200, 120, 60, 40, and $30 \AA$, cladded by $300-\AA$-thick $\operatorname{In}_{x} \mathrm{Ga}_{1-x} \mathrm{P}$ barriers (the In content in each sample is the same for both buffer and barriers). The outermost barrier is $500 \AA$ thick followed by a 40-Å-thick GaAs cap layer. All samples were characterized by double crystal x-ray diffraction for the (004) and (115) reflections. These results show that $\mathrm{InGaP}$ layers are fully strained and have the following In contents: $x=46.4,51.0$, and $45.0( \pm 0.1) \%$ for samples 1,2 , and 3 (strain conditions: $\epsilon=1.5 \times 10^{-3},-2.5 \times 10^{-3}$, and $1.9 \times 10^{-3}$, respectively). Continuous wave photoluminescence (PL) and PL excitation (PLE) measurements have been performed from 2 to $300 \mathrm{~K}$ by using the $514 \mathrm{~nm}$ line of an $\mathrm{Ar}^{+}$laser and an $\mathrm{Ar}^{+}$pumped Ti:sapphire laser, under low excitation regime, respectively.

Figure 1 summarizes the PL spectra measured for the three samples in the regions of interest. An increasing blue shift is observed in the QW PL lines from sample 2 to sample 3, as shown in Fig. 1(a). Transition energies without exciton correction have been also calculated by using our experimental results [PL shown in Fig. 1(b) and piezoreflectance not shown here] for the strained alloy band gap and the heavy-light hole splitting (about $-10,20$, and $-16 \mathrm{meV}$ for samples 1, 2, and 3, respectively). These calculations [marks in Fig. 1(a)] have been performed under the assumption of an increase of the conduction band gap offset from $Q_{c}=0.07$ (sample 2) to $Q_{c}=0.14$ (sample 1) and $Q_{c}=0.2$ (sample 3), in order to reproduce the experimental blue shift. This hypothesis seems reasonable; in fact a net increase of $Q_{c}$ with the $\mathrm{P}$ content in tensile strained $\mathrm{GaAsP} / \mathrm{GaAs}$ have been reported. ${ }^{9}$ At the same time, the estimated value $Q_{c}=0.14$ for sample 1 , closer to lattice match composition, is practically that deduced in Ref. 10. The energy shift of the InGaP excitonic line [Fig. 1(b)], from $1.927 \mathrm{eV}$ for sample 2 to $2.013 \mathrm{eV}$ for sample 3 , is in agreement with reported values for the compositional range used here. ${ }^{11-13}$ The low-energy

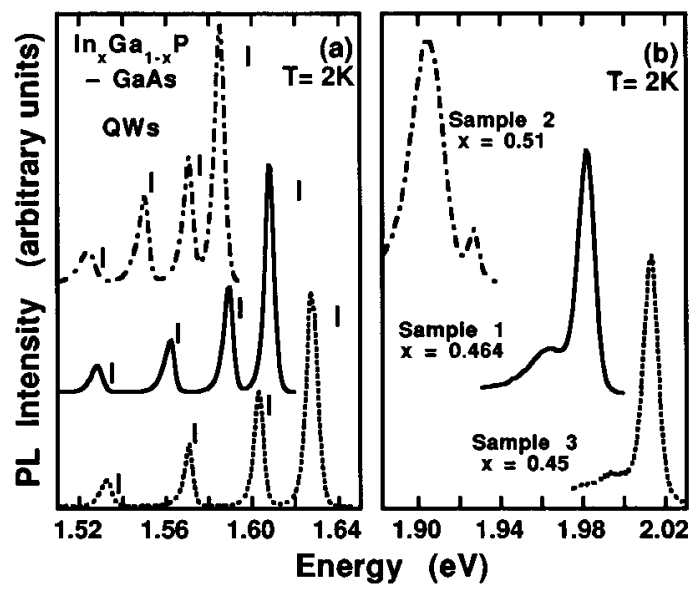

FIG. 1. PL spectra excited with an $\mathrm{Ar}^{+}$laser at $2 \mathrm{~K}$ for all samples in the regions of interest: (a) GaAs QWs and (b) InGaP barriers. In the latter case, the overall PL intensity is about two orders of magnitude lower than that measured for the 30-A-thick QWs. Marks added in (a) are calculated transition energies: $1.515 \mathrm{eV}+$ conduction + valence subband energies. 


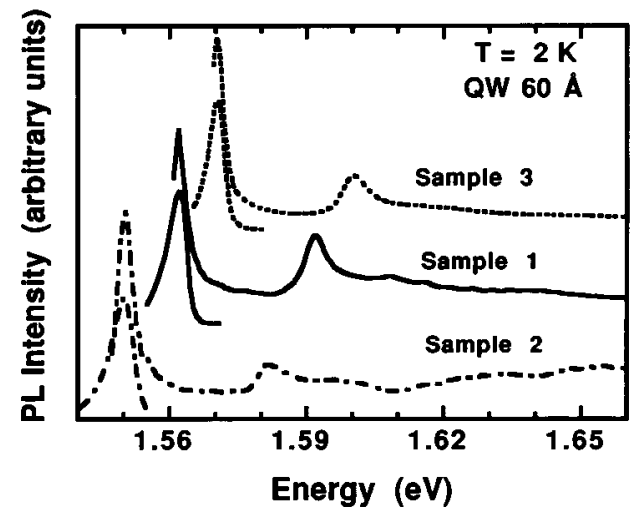

FIG. 2. PLE spectra corresponding to the $60 \AA$ QWs measured at $2 \mathrm{~K}$. PL spectra are also shown for comparison. Note that the linewidths of the fundamental optical transitions in PLE spectra are smaller than those of PL spectra.

peaks are attributed to acceptors, as discussed below. The PL linewidth for $\operatorname{In}_{x} \mathrm{Ga}_{1-x} \mathrm{P}$ layers range from 8 to $10 \mathrm{meV}$, of the same order than the best values achieved by other epitaxial growth techniques and different growth conditions. ${ }^{1,2,14,15}$ The PL linewidth for QWs ranges from 4 to 6 $\mathrm{meV}$. At the same time, a negligible Stokes shift (SS) is detected for the 60 - $\AA$-wide QWs, as shown in Fig. 2. The SS is also zero for the other quantum wells, except for the 30$\AA$-thick ones $(\mathrm{SS}=2-3 \mathrm{meV})$. The latter parameters, assessing the optical quality of the structures, are comparable or better than those reported in the literature. ${ }^{4-8}$

The optical quality is lower for the nominally $120-\AA$ wide QWs. In fact, a red shift is observed when PL is excited by infrared light, as shown in Fig. 3. This effect is also observed in samples 2 and 3, in which the red shift is stronger and weaker, respectively. Moreover, no emission is detected from the 200- $\AA$-thick QW (also the deepest in each structure) and the optical quality increases for outer QWs. It can be explained by a flattening of the InGaP free surfaces after the growth of the first GaAs layer $(200 \AA)$, being nearly total after the growth of the second one $(120 \AA)$. This flattening effect of a binary grown on a ternary has been also observed for the analogous system InP-InGaAs grown by ALMBE. ${ }^{16}$

Figure 4 shows the temperature variation of the PL integrated intensity for two QWs (60 and $30 \AA$ ) and InGaP lay-

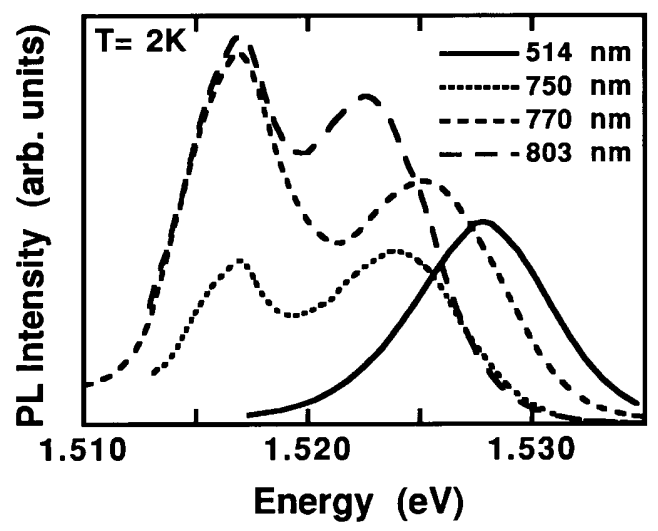

FIG. 3. PL spectra at $2 \mathrm{~K}$ from the $120 \AA \mathrm{QW}$ of sample 1 for different excitation wavelengths.

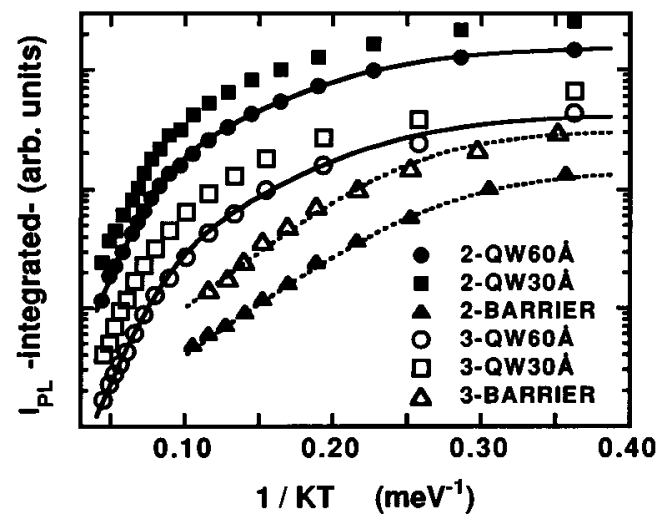

FIG. 4. Arrhenius plot of the relative PL-line integrated intensity for the 30and 60 - $\AA$-thick QWs and the InGaP barriers of samples 2 and 3 . Continuous lines are representative fits to Eq. (1).

ers of samples 2 and 3. The PL efficiency tends to be constant below $30 \mathrm{~K}$, similarly to the sample studied in Ref. 5 . However, the PL integrated intensity decreases by one order of magnitude between 30 and $90 \mathrm{~K}$ in that case, ${ }^{5}$ as compared to the observed reduction of a factor 4-5 in our QWs. As a matter of fact, similar dependence with temperature is observed in all QWs and even in the three samples, indicating the possibility of carrier losses inside the QW or at the interfaces, instead of the well known thermal emission of carriers to the outer barriers where they recombine nonradiatively. ${ }^{16-18}$

The lines fitting our experimental results (Fig. 4) have been obtained by using a classical thermal activation model $;^{17-19}$

$$
I_{P L}(T)=\frac{I_{0}}{1+\tau_{0} \Gamma_{1} e^{-E_{1} / K T}+\tau_{0} \Gamma_{2} e^{-E_{2} / K T}},
$$

where $\tau_{0}$ is the exciton radiative lifetime at low temperature $(\approx 1 \mathrm{~ns})$ and $1 / \Gamma_{1,2}$ the room-temperature nonradiative lifetimes for two possible carrier loss mechanisms. The fit of Eq. (1) to the experimental data for all QWs and samples gives the following values: $E_{1} \approx 16-20 \mathrm{meV}, \Gamma_{1}^{-1} \approx 20-30 \mathrm{ps}$, $E_{2} \approx 70-85 \mathrm{meV}, \Gamma_{2}^{-1} \approx 0.1-0.3 \mathrm{ps}$. The first mechanism could be associated to that observed for InGaP layers $\left(E_{1}\right.$ $\approx 20-26 \mathrm{meV}, \Gamma_{1}^{-1} \approx 2-4 \mathrm{ps}, \Gamma_{2}^{-1} \approx 0$ ), and thus it could be related to acceptor impurities located at the interfaces. In fact, the PL spectra associated to the alloy [Fig. 1(b)] has a low-energy contribution (18-22 meV below), that we assume due to $e-A^{0}$, as deduced from the temperature and power dependence of this emission line. The second nonradiative mechanism could be associated to acceptors inside the QWs or deeper impurity levels at the interfaces. Further improvement of the optical quality of InGaP/GaAs QWs can be expected in the future by optimizing growth conditions.

Figure 5 shows a qualitative dependence of the relative oscillator strength on the fundamental optical transition energy. The values shown in Fig. 5 have been obtained as follows:

the same portion of carriers is captured in each QW from those photogenerated in the half part of the two closer cladding barriers; 


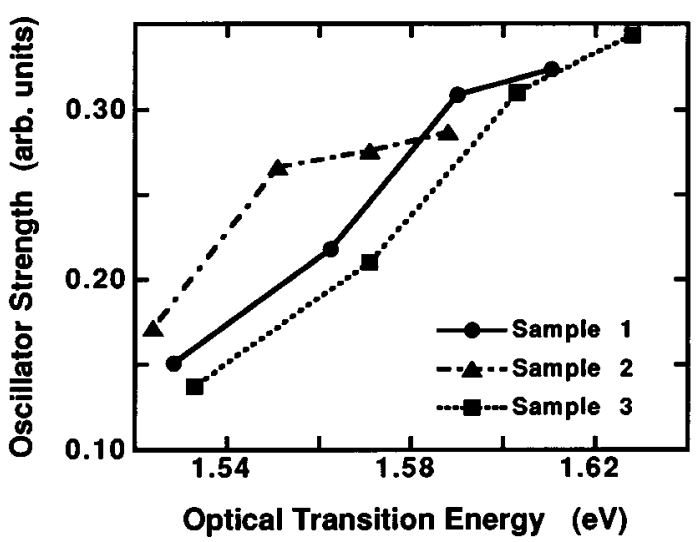

FIG. 5. Qualitative dependence of the optical transmission oscillator strength on the confinement energy in each sample.

(ii) the photon flux is reduced in each layer by the factor $\exp (-\alpha z), \alpha \approx 10^{5} \mathrm{~cm}^{-1}$ for GaAs and $\mathrm{InGaP}$ at $\lambda=514 \mathrm{~nm}$;

(iii) we calculate the ratio between $\int I_{P L}(E) d E$ for each QW [Fig. 1(a)] and the corresponding number of carriers photocreated; and

(iv) the four values obtained in each sample are finally normalized to the sum.

For thick QWs (60 and $120 \AA$ ), the oscillator strength seems to increase from sample 3 (tensile strain) to sample 2 (compressive strain), due to the dependence of this parameter on the effective mass. The opposite tendence is observed for thinner QWs. On the one hand, a saturation of the oscillator strength is observed for sample 2, which is coherent with the low estimated value for $Q_{c}$, inducing a fast saturation of the electron subband energy and the associated spread of the electron wave function throughout the barriers. ${ }^{20}$ On the other hand, the oscillator strength increases by a factor 2.5 from the $120 \AA$ to the $30 \AA$ QW in sample 3 , which is similar to the observed and calculated increase in the $\mathrm{AlGaAs} / \mathrm{GaAs}$ system. ${ }^{21}$

In summary, InGaP/GaAs quantum wells of good optical quality have been grown successfully by ALMBE. In the indium content range from 0.51 to 0.45 (compressive to tensile strained barriers) we have observed a gain in oscillator strength for thin QWs and vice versa for thicker ones. Simultaneously, an important blue shift of the confinement energies has been shown, which has been explained assuming an increase of the conduction band gap offset. Acceptor impurities located in the GaAs QWs and their interfaces with InGaP seems to be responsible of the temperature quenching of the luminescence.

One of us (J.M.-P.) thanks the European Community for Human Capital and Mobility financial support, and also the Spanish DGICYT (project No. PB93-0687). The "Laboratoire de Physique de la Matière Condensée" is a "Laboratoire associé au CNRS (URA 1437) et aux Universités Paris VI et VII."' Thanks are also given to Gaspar Armelles from the CNM for assistance in piezoreflectivity experiments.

${ }^{1}$ M. C. DeLong, D. J. Mowbray, R. A. Hogg, M. S. Skolnick, M. Hopkinson, J. P. R. David, and P. C. Taylor, J. Appl. Phys. 73, 5163 (1993).

${ }^{2}$ G. S. Horner, A. Mascarenhas, R. G. Alonso, S. Froyen, K. A. Bertness, and J. M. Olson, Phys. Rev. B 49, 1727 (1994).

${ }^{3}$ D. M. Follstaedt, R. P. Schneider, Jr., and E. D. Jones, J. Appl. Phys. 77, 3077 (1995).

${ }^{4}$ M. J. Hafich, J. H. Quigley, R. E. Owens, G. Y. Robinson, Du Li, and N. Otsuka, Appl. Phys. Lett. 54, 2686 (1989).

${ }^{5}$ J.Ch. García, Ph. Maurel, Ph. Bove, and J. P. Hirtz, J. Appl. Phys. 30, 1186 (1991).

${ }^{6}$ F. Omnes and M. Razeghi, Appl. Phys. Lett. 59, 1034 (1991).

${ }^{7}$ C. Jelen, S. Slivken, X. G. He, M. Razeghi, and S. Shastry, J. Vac. Sci. Technol. B 12, 1113 (1994).

${ }^{8}$ D. J. Mowbray, O. P. Kowalski, M. S. Skolnick, M. C. DeLong, M. Hopkinson, J. P. R. David, and A. G. Cullis, J. Appl. Phys. 75, 2029 (1994).

${ }^{9}$ X. Zhang, K. Onabe, H. Yaguchi, Y. Shiraki, and R. Ito, Jpn. J. Appl. Phys. 32, L375 (1991); W. Shan, S. J. Hwang, J. J. Song, H. Q. Hou, and C. W. Tu, Appl. Phys. Lett. 62, 2078 (1993).

${ }^{10}$ J. Chen, J. R. Sites, I. L. Spain, M. J. Hafich, and G. Y. Robinson, Appl. Phys. Lett. 58, 744 (1991).

${ }^{11}$ D. Moroni, E. Dupont-Nivet, J. P. André, J. N. Patillon, and C. Delalande, J. Appl. Phys. 63, 5188 (1988).

${ }^{12}$ K. Ozasa, M. Yuri, S. Tanaka, and H. Matsunami, J. Appl. Phys. 68, 107 (1990).

${ }^{13}$ M. Zachau and W. T. Masselink, Appl. Phys. Lett. 60, 2098 (1992),

${ }^{14}$ C. T. H. F. Liedenbaum, A. Valster, A. L. G. J. Severens, and G. W. 't Hooft, Appl. Phys. Lett. 57, 2698 (1990).

${ }^{15}$ R. P. Schneider, Jr., E. D. Jones, J. A. Lott, and R. P. Bryan, J. Appl. Phys. 72, 5397 (1992).

${ }^{16} \mathrm{~L}$. González and Y. González (unpublished).

${ }^{17}$ M. Gurioli, J. Martínez-Pastor, M. Colocci, C. Deparis, B. Chastaingt, and J. Massies, Phys. Rev. B 46, 6922 (1992).

${ }^{18}$ M. Vening, D. J. Dunstan, and K. P. Homewood, Phys. Rev. B 48, 2412 (1993).

${ }^{19}$ E. M. Daly, T. J. Glynn, J. D. Lambkin, L. Considine, and S. Walsh, Phys. Rev. B 52, 4696 (1995).

${ }^{20}$ J. Martínez-Pastor, M. Gurioli, M. Colocci, C. Deparis, B. Chastaingt, and J. Massies, Phys. Rev. B 46, 2239 (1992).

${ }^{21}$ L. C. Andreani and A. Pasquarello, Phys. Rev. B 42, 8928 (1990). 